\title{
Designing of Novel 6(H)-1,3,4-Thiadiazine Derivatives as MMP12 Inhibitors: A MLR and Docking Approach
}

\author{
Ajeet, Laxmi Tripathi, Praveen Kumar ${ }^{*}$ \\ Department of Pharmaceutical Chemistry, S. D. College of Pharmacy and Vocational Studies, Muzaffarnagar, India \\ *Corresponding author: praveensha77@gmail.com
}

Received February 28, 2013; Revised March 30, 2013; Accepted April 03, 2013

\begin{abstract}
Here 6(H)-1,3,4-thiadiazine analogues have been used to correlate the inhibiting constant with the eccentric connectivity index (ECI), fragment complexity (FC), McGowan volume (MG) and topological polar surface area (TPSA) for studying the quantitative structure activity relationship (QSAR). Correlation may be an adequate predictive model which can help to provide guidance in designing and subsequently yielding greatly specific compounds that may have reduced side effects and improved pharmacological activities. The literature survey revealed that there are so many models available for MMP-1, MMP-2, MMP-3, MMP-7, MMP-8, MMP-9 and MMP-13, but QSAR model in respect of MMP-12 inhibition was necessity. We have used multiple linear regression (MLR) for developing QSAR model. For the validation of the developed QSAR model, statistical analysis such as cross validation test, standard deviation, quality factor, fischers test, root mean square deviation (RMSD), variance; and internal validation such as Y-randomization test have been performed and all the tests validated this QSAR model with fraction of variance $\mathrm{r}^{2}=0.9364$ and LOO-CV variance $\mathrm{q}^{2}=0.9146$. Thirteen novel 6(H)-1,3,4-thiadiazine analogues have been designed and their inhibiting constant have been calculated with the developed QSAR model. It was found that the calculated inhibiting constant of these analogues were within the same range as of the training set. Further, these $6(\mathrm{H})-1,3,4$-thiadiazine analogues have been docked with the catalytic domain of human matrix metalloproteinase (MMP12) which shows better docking score as compared to the (Nhydroxy-2-(N-hydroxyethyl)biphenyl-4-ylsulfonamido) acetamide, a MMP12 inhibitor. The results suggested that the designed novel 6(H)-1,3,4-thiadiazine analogues could be developed as a good MMP12 inhibitors.
\end{abstract}

Keywords: 6(H)-1,3,4-thiadiazine analogues, QSAR, MLR, docking, MMP12 inhibitors

\section{Introduction}

Matrix Metalloproteinase (MMPs) is a family of calcium dependent zinc containing endopeptidase, which is responsible for the remodeling of tissue and extracellular matrix degradation, including gelatin, matrix glycoprotein, proteoglycan etc. These are excreted by a variety of connective tissue and some pro-inflammatory cells like lymphocytes, osteoblasts, macrophages, endothelial cells, neutrophils, and fibroblasts. MMPs have been considered as a target for cancer therapy and a number of natural and synthetic MMPs inhibitors have been identified as cytostatic and anti-angiogenic agents. MMPs consists of four domains, these are hinge region, $\mathrm{N}$-terminal pro-domain, catalytic domain, and C-terminal hemopexin-like domain. It may be responsible for interaction with tissue inhibitors of MMPs as well as for the macromolecular substrate recognition [1]. Matrix metalloproteinase-12 (MMP-12) also known as macrophage metalloelastase (MME) or macrophage elastase (ME) is an enzyme that in humans is encoded by the MMP12 gene. The literature review revealed that inhibitory activity of various compounds with the MMP-1, MMP-2, MMP-3, MMP-7, MMP-8, MMP-9 and MMP-13 has been correlated with hydrophobicity, Kier's first order valence molecular connectivity index, electrotopological state indices of the atom and polarizability. Hydrophobicity is the most important descriptor and have been found to describe the inhibitory activity of $6(\mathrm{H})$ 1,3,4-thiadiazine derivatives [2]. Further, it was concluded that development of a QSAR model dealing with MMP-12 inhibition was necessary.

The QSAR studies are perfect tool for understanding the drug design process in terms of their chemicalpharmacological activity interaction. QSAR studies can focus on mechanism of action of ligands with human, bacteria, virus, membranes, enzymes etc. The QSAR methodology comprises of computationally derived descriptors to correlate with pharmacological activities. These descriptors are principally of four types such as electronic, stearic, hydrophobic and topological indices [3]. The descriptors used for developing the QSAR model are eccentric connectivity index (ECI) [4], fragment complexity (FC) [5], McGowan volume (MG) [6] and topological polar surface area (TPSA) [7]. Rational drug design helps to facilitate and fasten the drug designing process, which involves various methods to identify novel compound, out of them one method is the docking of molecules with the receptor [8].

In the present study, we developed a QSAR model on a series of $6(\mathrm{H})-1,3,4$-thiadiazine analogues with respect to their MMP12 inhibition. Further, these 6(H)-1,3,4- 
thiadiazine analogues have been docked with the catalytic domain of MMP12.

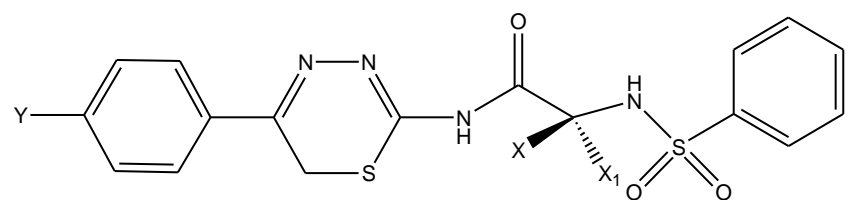

Figure 1.Structure of 6(H)-1,3,4-thiadiazine used for model development

\section{Materials and Method}

All the bioactivity values and information about 2D structure of $6(\mathrm{H})$-1,3,4-thiadiazine derivatives (Figure 1) were taken from literature [1]. $\mathrm{IC}_{50}$ is referred as the molar concentration of a compound that inhibits $50 \%$ growth of bacteria [3]; $\log 1 / \mathrm{K}_{\mathrm{i}}\left(\mathrm{K}_{\mathrm{i}}\right.$ is inhibition constant $)$ is subsequent variable that comprises the bioactivity parameter for the QSAR model. In order to calculate the 2D molecular descriptors, PaDEL descriptor software [9] which incorporate CDK library for descriptor calculation have been used after optimitizing the $6(\mathrm{H})-1,3,4-$ thiadiazine derivatives. For the development of QSAR model, MLR [3] has been employed and all were validated through statistics. The novel $6(\mathrm{H})-1,3,4$-thiadiazine molecules have been designed and optimized through ChemDraw Ultra 7.0.and their bioactivity values have been calculated from developed QSAR model by putting the descriptor values in the QSAR equation. The docking studies [8] of novel 6(H)-1,3,4-thiadiazine have been performed with the Molegro Virtual Docker (Free trial version).

\subsection{Statistical Parameters}

\subsubsection{Fraction of Variance $\left(\mathbf{R}^{2}\right)$}

The value of fraction of variance may vary between 0 (means model without explanatory power) and 1 (means perfect model). QSAR model having $\mathrm{r}^{2}>0.6$ will only be considered for validation [3].

\subsubsection{Cross-validation Test $\left(\mathbf{q}^{2}\right)$}

A QSAR model must have $\mathrm{q}^{2}>0.5$ for the predictive ability [3].

\subsubsection{Standard Deviation (s)}

The smaller s value is always required for the predictive QSAR model.
The difference between $\mathrm{r}^{2}$ and $\mathrm{q}^{2}$ should never be exceeding by 0.3 . A large difference suggests the following: presence of outliers, over-fitted model, and presence of irrelevant variables in data [3].

\subsubsection{Quality Factor (Q)}

Overfitting and chance correlation, due to excess number of descriptors, can be detected by $Q$ value. Positive value for this QSAR model suggests its high predictive power and lack of overfitting.

\subsubsection{Fischer Statistics (F)}

The F value of QSAR model was compared with their literature value at $95 \%$ level.

\subsection{Model Validation}

The QSAR model validation was carried with statistical analysis and with internal validation.

\subsection{Docking Studies}

The designed novel $6(\mathrm{H})-1,3,4$-thiadiazine derivatives were docked into catalytic domain of humanMMP12. For this study, X-ray crystal structure of human MMP12 was taken from protein data bank with PDB id 3N2V.

\subsubsection{Determination of Binding Site}

The catalytic sites of human MMP12 have been found by DETECT CAVITIES under the PREPARATION option of Molegro Virtual Docker (Free trial version) which detects 5 cavities by default with a $10 \AA$ resolution.

\section{Results and Discussion}

From the data (Table 1), QSAR equation 1 have been developed and $95 \%$ confidence intervals are given in parantheses as follows

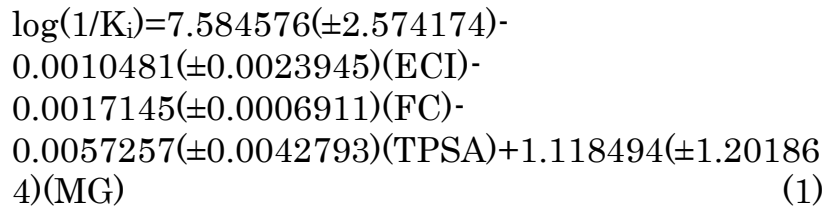

\subsection{Validation of QSAR Model}

A quantitative assessment of model robustness has been performed through model validation. All the statistical results of model validation have been given (Table 2).

\subsection{4. $r^{2}-q^{2}<0.3$}

Table 1. Descriptors used to derive QSAR equation along with bioactivities for the inhibition by $6(\mathrm{H})$-1,3,4-thiadiazine analogues with substituents

\begin{tabular}{|c|c|c|c|c|c|c|c|c|c|}
\hline \multicolumn{3}{|c|}{ Substituents } & \multicolumn{3}{|c|}{$\log 1 / K_{i}$} & \multirow[t]{2}{*}{ ECI } & \multirow[t]{2}{*}{$\mathrm{FC}$} & \multirow[t]{2}{*}{ TPSA } & \multirow[t]{2}{*}{$\mathrm{MG}$} \\
\hline $\mathrm{X}$ & $\mathrm{X}_{1}$ & $\bar{Y}$ & Obs. & Pred. & Diff. & & & & \\
\hline $\mathrm{CH}_{3}$ & $\mathrm{H}$ & $\mathrm{F}$ & 6.46 & 6.499902 & -0.0399 & 775 & 1744.09 & 106.5238 & 2.9752 \\
\hline $\mathrm{H}$ & $\mathrm{CH}_{3}$ & $\mathrm{Cl}$ & 6.28 & 6.352982 & -0.07298 & 825 & 1939.1 & 116.0725 & 3.2385 \\
\hline $\mathrm{CH}_{3}$ & $\mathrm{H}$ & $\mathrm{Cl}$ & 6.49 & 6.429318 & 0.060682 & 825 & 1939.1 & 102.7402 & 3.2385 \\
\hline $\mathrm{CH}_{3}$ & $\mathrm{H}$ & $\mathrm{Br}$ & 6.47 & 6.525846 & -0.05585 & 911 & 1570.12 & 139.563 & 3.0283 \\
\hline $\mathrm{H}$ & $\mathrm{CH}_{3}$ & $\mathrm{CN}$ & 6.43 & 6.316241 & 0.113759 & 841 & 1789.1 & 124.5081 & 3.0339 \\
\hline $\mathrm{H}$ & $\mathrm{CH}_{3}$ & $\mathrm{CH}_{3}$ & 6.96 & 6.986579 & -0.02658 & 775 & 1453.1 & 105.044 & 2.9567 \\
\hline $\mathrm{CH}_{3}$ & $\mathrm{H}$ & $\mathrm{CH}_{3}$ & 7.05 & 6.989891 & 0.060109 & 775 & 1453.1 & 104.4655 & 2.9567 \\
\hline $\mathrm{H}$ & $\mathrm{CH}_{3}$ & $\mathrm{NO}_{2}$ & 6.55 & 6.542078 & 0.007922 & 841 & 1492.1 & 165.2268 & 2.989 \\
\hline $\mathrm{H}$ & $\mathrm{CH}_{3}$ & $\mathrm{CF}_{3}$ & 6.52 & 6.517286 & 0.002714 & 841 & 1492.1 & 169.5568 & 2.989 \\
\hline $\mathrm{H}$ & $\mathrm{CH}_{3}$ & $\mathrm{OCH}_{3}$ & 6.47 & 6.519877 & -0.04988 & 775 & 1744.09 & 103.035 & 2.9752 \\
\hline
\end{tabular}


Table 2. Results of statistical validation

\begin{tabular}{cccccccc}
\hline $\mathrm{r}^{2}$ & $\mathrm{q}^{2}$ & $\mathrm{~s}$ & $\mathrm{r}^{2}-\mathrm{q}^{2}<0.3$ & $\mathrm{Q}$ & RMSD & Variance & $\mathrm{F}$ \\
\hline 0.9364 & 0.9146 & 0.2318 & 0.0218 & 4.1747 & 0.018313 & 0.006708 & 18.4041 \\
\hline
\end{tabular}

\subsection{Internal Validation}

Y-Randomization Test:, This technique is used to establish the QSAR model robustness. For this test, the dependent variable vector is randomly shuffled, and a new
QSAR model is developed using the unchanged independent variable. This process was repeated for five times. The statistical data of $\mathrm{r}^{2}$ for five runs are given (Table 3). The values $r^{2}<0.6$ in $Y$-randomization test confirm the robustness of this QSAR model [3].

Table 3 Results of internal validation: Y-randomization test (5 runs)

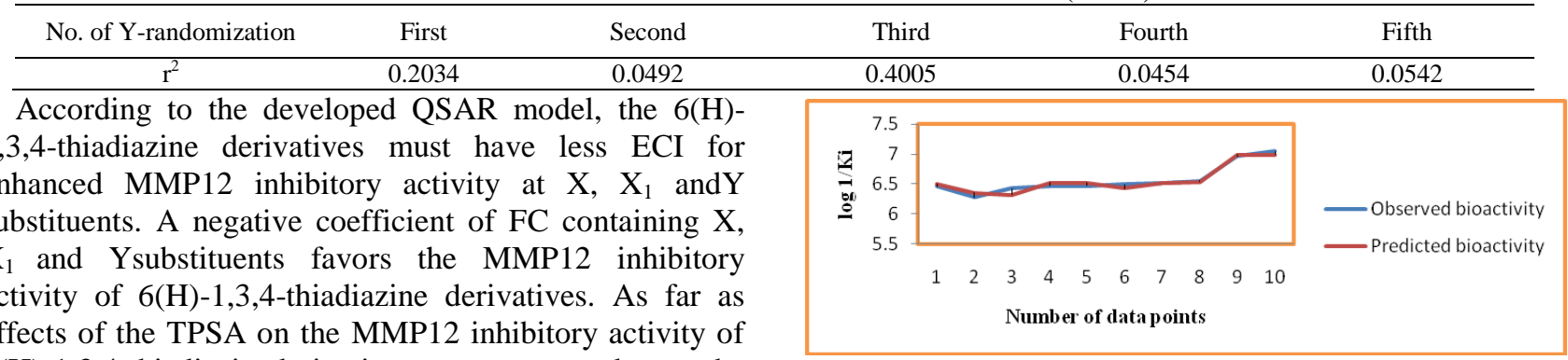

$6(\mathrm{H})-1,3,4$-thiadiazinederivatives concerned, the developed QSAR model suggested that an decrement in TPSA at substituents $\mathrm{X}, \mathrm{X}_{1}$ and $\mathrm{Y}$ will definitely be favorable to the activity; along with positive McGowan volume, as discussed by Verma and Hansch, $2010^{[3]}$. A comparison (MLR plots) of observed values and predicted values of $\log 1 /$ Kifor $6(\mathrm{H})$-1,3,4-thiadiazine derivatives used for development of QSAR equation is shown in Figure 2 and multiple linear graph is shown in Figure 3.

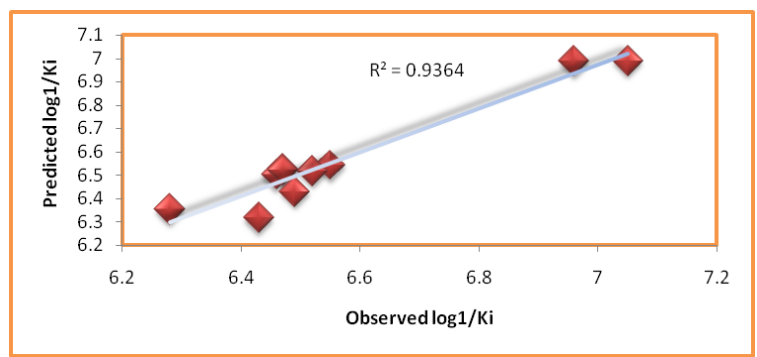

Figure 2. Multiple linear regression plot for QSAR study
Figure 3. Multiple linear graph between Number of data points and bioactivities

\subsection{Designing and Optimization of Novel 6(H)-1,3,4-thiadiazine Derivatives}

The novel 6(H)-1,3,4-thiadiazine derivatives were designed and their energy minimization for highest stability was performed. Details of substituents, calculated descriptor values and predicted bioactivity through derived QSAR model have been given (Table 4).

\subsection{Docking Results}

\subsubsection{Binding Site Analysis}

The experimental analysis of binding site shows that Leu 214, Tyr 240, Glu 219, Leu 181, Ala 182, Ile 180, His 228, Pro 238, Phe 237, Val 235, His 218 could be the catalytic site residue present in the structure of human MMP12.

Table 4. Results of QSAR studies on novel 6(H)-1,3,4-thiadiazine derivatives

\begin{tabular}{|c|c|c|c|c|c|c|c|c|}
\hline & \multicolumn{3}{|c|}{ Substituents } & \multirow{2}{*}{$\begin{array}{l}\log 1 / K_{\mathrm{i}} \\
\text { Predicted }\end{array}$} & \multirow{2}{*}{ ECI } & \multirow{2}{*}{$\mathrm{FC}$} & \multirow{2}{*}{ TPSA } & \multirow{2}{*}{ MG } \\
\hline & $\mathrm{X}$ & $\mathrm{X}_{1}$ & $\mathrm{Y}$ & & & & & \\
\hline THZ2 & $\mathrm{H}$ & $\mathrm{NH}_{2}$ & $\mathrm{Cl}$ & 6.426937119 & 775 & 1645.1 & 140.8755 & 2.9341 \\
\hline THZ3 & $\mathrm{NH}_{2}$ & $\mathrm{H}$ & $\mathrm{Cl}$ & 6.455465196 & 775 & 1548.11 & 156.9069 & 2.893 \\
\hline THZ4 & $\mathrm{NH}_{2}$ & $\mathrm{H}$ & $\mathrm{Br}$ & 6.835229491 & 876 & 1434.13 & 120.7563 & 2.9674 \\
\hline THZ5 & $\mathrm{H}$ & $\mathrm{NH}_{2}$ & $\mathrm{CN}$ & 6.551398878 & 911 & 1471.13 & 156.7094 & 2.9872 \\
\hline THZ6 & $\mathrm{H}$ & $\mathrm{CH}_{3}$ & $\mathrm{NH}_{2}$ & 6.327371314 & 841 & 1688.11 & 144.7713 & 2.9928 \\
\hline THZ7 & $\mathrm{H}$ & $\mathrm{NH}_{2}$ & $\mathrm{CH}_{3}$ & 7.041056927 & 775 & 1360.11 & 115.3421 & 2.9156 \\
\hline THZ8 & $\mathrm{H}$ & $\mathrm{NH}_{2}$ & $\mathrm{NH}_{2}$ & 7.014682572 & 775 & 1360.11 & 119.9484 & 2.9156 \\
\hline THZ9 & $\mathrm{NH}_{2}$ & $\mathrm{H}$ & $\mathrm{CH}_{3}$ & 7.073678417 & 775 & 1360.11 & 119.9199 & 2.9682 \\
\hline THZ10 & $\mathrm{CH}_{3}$ & $\mathrm{H}$ & $\mathrm{NH}_{2}$ & 6.584272664 & 841 & 1397.11 & 178.269 & 2.9479 \\
\hline THZ11 & $\mathrm{NH}_{2}$ & $\mathrm{H}$ & $\mathrm{NH}_{2}$ & 6.43287249 & 775 & 1645.1 & 139.8389 & 2.9341 \\
\hline THZ12 & $\mathrm{H}$ & $\mathrm{NH}_{2}$ & $\mathrm{NO}_{2}$ & 6.542596066 & 775 & 1645.1 & 120.6755 & 2.9341 \\
\hline THZ13 & $\mathrm{H}$ & $\mathrm{NH}_{2}$ & $\mathrm{CF}_{3}$ & 6.462036652 & 775 & 1548.11 & 155.7592 & 2.893 \\
\hline THZ14 & $\mathrm{H}$ & $\mathrm{NH}_{2}$ & $\mathrm{OCH}_{3}$ & 6.543720539 & 775 & 1645.1 & 120.4791 & 2.9341 \\
\hline
\end{tabular}

\subsubsection{Docking Studies of Novel 6(H)-1,3,4-thiadiazine Derivatives with Human MMP12}

Docking studies showed that derivatives THZ2-14 were docked in the same binding site as docked by (N-hydroxy2-(N-hydroxyethyl)biphenyl-4-ylsulfonamido) acetamide; a pre-existing human MMP12 inhibitor. The proteinligand interaction energy of novel inhibitors were given by Pose organizer option in Molegro virtual docker (Free trial version) for 5 different poses of novel inhibitors. The best pose interaction energy was found to be as -179.676 , $180.116,-180.162,-189.003,-182.567, \quad-186.026$, $175.835,-175.277,-174.593,-176.54,-117.702,-186.236$ and -190.092 . Here, negative values for interaction energy would reflect the positive docking approach. Number of hydrogen bonds and other binding details with cavities (Table 5) and docking images (Table 6) are given. 
Table 5. Docking results of novel $6(\mathrm{H})-1,3,4$-thiadiazine derivatives

\begin{tabular}{|c|c|c|c|c|c|c|c|}
\hline \multirow{3}{*}{ Novel molecules } & \multirow{3}{*}{$\begin{array}{l}\text { E-Inter. (ligand- } \\
\text { Protein) KJ/mol }\end{array}$} & \multirow{3}{*}{ H-bonds } & \multicolumn{5}{|c|}{ Binding description } \\
\hline & & & \multicolumn{2}{|c|}{ Ligand } & \multicolumn{3}{|c|}{ Receptor } \\
\hline & & & Element & Atom ID & Residue & Element & Atom ID \\
\hline \multirow{6}{*}{ THZ2 } & \multirow{6}{*}{-179.676} & \multirow{6}{*}{6} & $\mathrm{~N}$ & 15 & His 218 & $\mathrm{~N}$ & 883 \\
\hline & & & $\mathrm{N}$ & 16 & His 218 & $\mathrm{~N}$ & 883 \\
\hline & & & $\mathrm{N}$ & 7 & Ala 182 & $\mathrm{O}$ & 616 \\
\hline & & & $\mathrm{N}$ & 27 & Ala 182 & $\mathrm{O}$ & 616 \\
\hline & & & $\mathrm{N}$ & 27 & Glu 219 & $\mathrm{O}$ & 894 \\
\hline & & & $\mathrm{N}$ & 7 & Glu 219 & $\mathrm{O}$ & 894 \\
\hline \multirow{3}{*}{ THZ3 } & \multirow{3}{*}{-180.116} & \multirow{3}{*}{3} & $\mathrm{~N}$ & 16 & His 218 & $\mathrm{~N}$ & 883 \\
\hline & & & $\mathrm{N}$ & 7 & Ala 182 & $\mathrm{O}$ & 616 \\
\hline & & & $\mathrm{N}$ & 14 & Glu 219 & $\mathrm{O}$ & 894 \\
\hline \multirow{2}{*}{ THZ4 } & \multirow{2}{*}{-180.162} & \multirow{2}{*}{2} & $\mathrm{~N}$ & 14 & Lys 241 & $\mathrm{O}$ & 1054 \\
\hline & & & $\mathrm{N}$ & 16 & Tyr 240 & $\mathrm{~N}$ & 1039 \\
\hline \multirow{5}{*}{ THZ5 } & & & $\mathrm{N}$ & 15 & His 218 & $\mathrm{~N}$ & 883 \\
\hline & & & $\mathrm{H}$ & 34 & Glu 219 & $\mathrm{O}$ & 894 \\
\hline & -189.003 & 5 & $\mathrm{H}$ & 34 & His 218 & $\mathrm{~N}$ & 886 \\
\hline & & & $\mathrm{H}$ & 34 & Ala 182 & $\mathrm{O}$ & 616 \\
\hline & & & $\mathrm{N}$ & 28 & Glu 219 & $\mathrm{O}$ & 894 \\
\hline & & & $\mathrm{N}$ & 26 & Lys 241 & $\mathrm{O}$ & 1054 \\
\hline & & & $\mathrm{N}$ & 16 & Tyr 240 & $\mathrm{~N}$ & 1039 \\
\hline & & & $\mathrm{N}$ & 16 & His 218 & $\mathrm{~N}$ & 883 \\
\hline & & & $\mathrm{N}$ & 15 & His 218 & $\mathrm{~N}$ & 883 \\
\hline THZ6 & -182.567 & 8 & $\mathrm{~N}$ & 12 & His 218 & $\mathrm{~N}$ & 886 \\
\hline & & & $\mathrm{O}$ & 9 & Ile 180 & $\mathrm{~N}$ & 597 \\
\hline & & & $\mathrm{O}$ & 8 & Ala 182 & $\mathrm{~N}$ & 613 \\
\hline & & & $\mathrm{O}$ & 8 & Leu 181 & $\mathrm{~N}$ & 605 \\
\hline & & & $\mathrm{N}$ & 7 & Ala 182 & $\mathrm{O}$ & 616 \\
\hline & & & $\mathrm{N}$ & 7 & Glu 219 & $\mathrm{O}$ & 895 \\
\hline THZ7 & -186.026 & 5 & $\mathrm{~N}$ & 7 & His 218 & $\mathrm{~N}$ & 886 \\
\hline & & & $\mathrm{N}$ & 27 & Glu 219 & $\mathrm{O}$ & 894 \\
\hline & & & $\mathrm{N}$ & 15 & His 216 & $\mathrm{~N}$ & 883 \\
\hline & & & $\mathrm{N}$ & 26 & Gly 179 & $\mathrm{O}$ & 596 \\
\hline & & & $\mathrm{N}$ & 16 & Tyr 240 & $\mathrm{~N}$ & 1039 \\
\hline THZ8 & -175.835 & 5 & $\mathrm{O}$ & 13 & Lys 241 & $\mathrm{~N}$ & 1051 \\
\hline & & & $\mathrm{O}$ & 8 & Val 235 & $\mathrm{C}$ & 1000 \\
\hline & & & $\mathrm{N}$ & 27 & Leu 214 & $\mathrm{O}$ & 853 \\
\hline & & & $\mathrm{N}$ & 14 & Ala 182 & $\mathrm{O}$ & 616 \\
\hline ТHZO & 175277 & 4 & $\mathrm{~N}$ & 16 & His 218 & $\mathrm{~N}$ & 883 \\
\hline THZ9 & -175.217 & 4 & $\mathrm{~N}$ & 17 & His 218 & $\mathrm{~N}$ & 883 \\
\hline & & & $\mathrm{N}$ & 17 & Tyr 240 & $\mathrm{~N}$ & 1039 \\
\hline & & & $\mathrm{N}$ & 27 & Ala 182 & $\mathrm{O}$ & 616 \\
\hline THZ10 & -174.593 & 3 & $\mathrm{O}$ & 13 & Lys 241 & $\mathrm{~N}$ & 1051 \\
\hline & & & $\mathrm{O}$ & 8 & Val 235 & $\mathrm{~N}$ & 999 \\
\hline & & & $\mathrm{N}$ & 27 & Ala 182 & $\mathrm{O}$ & 616 \\
\hline & & & $\mathrm{N}$ & 27 & Glu 219 & $\mathrm{O}$ & 894 \\
\hline THZ11 & -176.54 & 4 & $\mathrm{~N}$ & 12 & Lys 241 & $\mathrm{O}$ & 1054 \\
\hline & & & $\mathrm{N}$ & 14 & Lys 241 & $\mathrm{O}$ & 1054 \\
\hline THZ12 & -117702 & 2 & $\mathrm{O}$ & 40 & His 218 & $\mathrm{~N}$ & 883 \\
\hline 1HZ12 & $-11 / . / 02$ & 2 & $\mathrm{O}$ & 33 & Tyr 240 & $\mathrm{~N}$ & 1039 \\
\hline & & & $\mathrm{O}$ & 8 & Val 235 & $\mathrm{~N}$ & 999 \\
\hline & & & $\mathrm{N}$ & 7 & Lys 241 & $\mathrm{O}$ & 1054 \\
\hline THZ13 & -186.236 & 5 & $\mathrm{O}$ & 13 & Lys 241 & $\mathrm{~N}$ & 1051 \\
\hline & & & $\mathrm{N}$ & 16 & His 218 & $\mathrm{~N}$ & 883 \\
\hline & & & $\mathrm{N}$ & 16 & Tyr 240 & $\mathrm{~N}$ & 1039 \\
\hline & & & $\mathrm{N}$ & 15 & His 218 & $\mathrm{~N}$ & 883 \\
\hline TH714 & -190092 & 4 & $\mathrm{~N}$ & 28 & Glu 219 & $\mathrm{O}$ & 894 \\
\hline THZ14 & -190.092 & 4 & $\mathrm{~N}$ & 7 & Glu 219 & $\mathrm{O}$ & 894 \\
\hline & & & $\mathrm{N}$ & 7 & Ala 182 & $\mathrm{O}$ & 616 \\
\hline
\end{tabular}




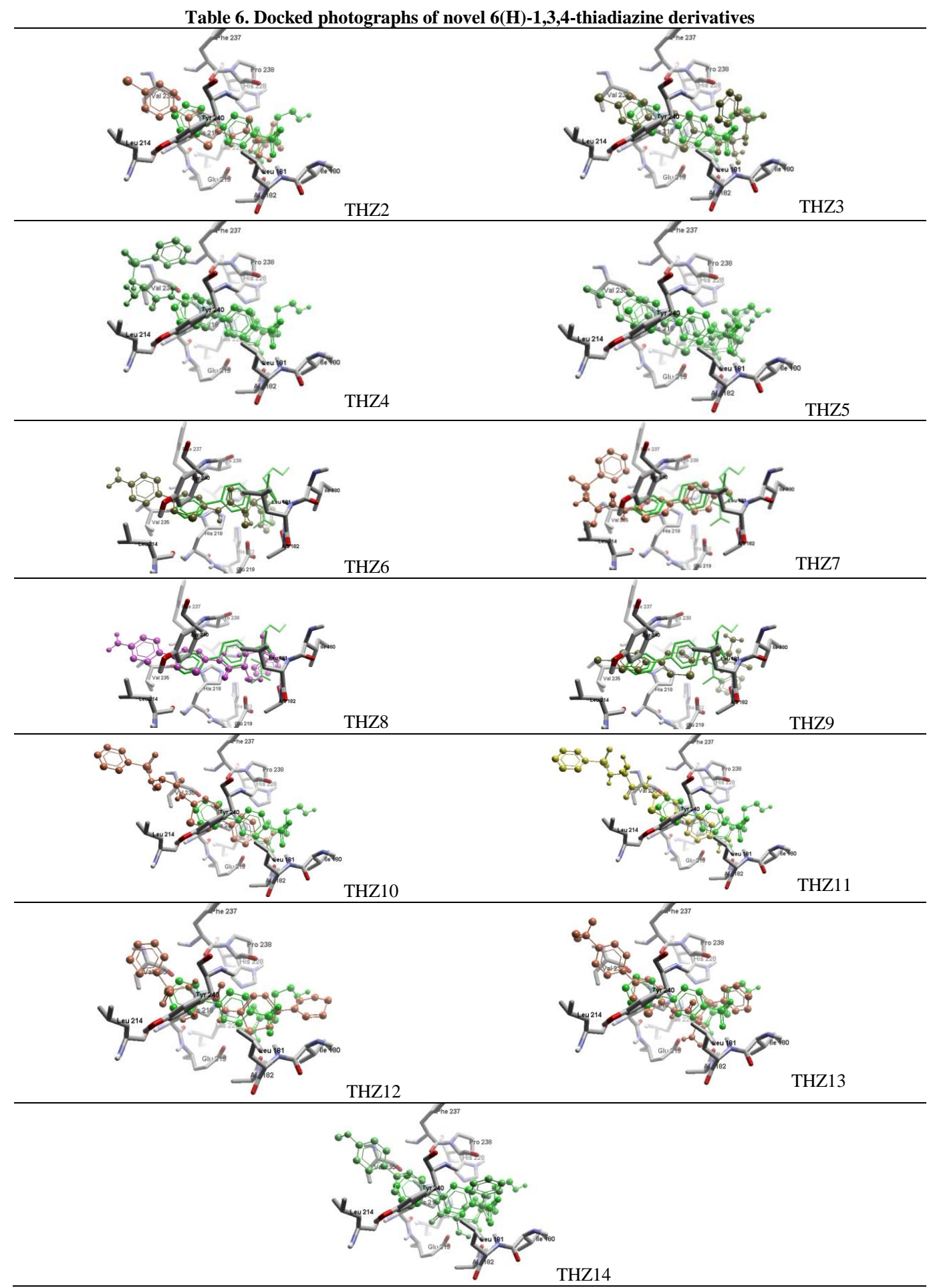

\subsection{Comparison of Docking Results with Pre- existing Human MMP-12 Inhibitor (N- Hydroxy-2-(N-Hydroxyethyl)Biphenyl-4- Ylsulfonamido)Acetamide}

Docking analysis of (N-hydroxy-2-(N-hydroxyethyl) biphenyl-4-ylsulfonamido)acetamide with the MMP-12 revealed that interacting residues (amino acids) were His 222, His 228, Pro 238, Phe 237, Val 235, His 218, Thr 239, His 183, Glu 219, Ala 182, Thr 215, Tyr 240 and Leu 214 (Figure 4). These results were similar to the docking analysis of novel designed 6(H)-1,3,4-thiadiazine derivatives.

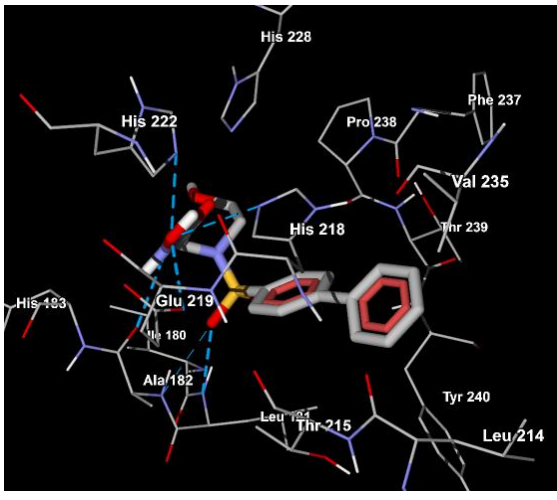

Figure 4. Docking image of (N-hydroxy-2-(N-hydroxyethyl)biphenyl-4ylsulfonamido) acetamide with the MMP-12 showing interacting amino acids 
On docking analysis, the docking images of all novel 6(H)-1,3,4-thiadiazine derivatives were found to be superimposed with the (N-hydroxy-2-(Nhydroxyethyl)biphenyl-4-ylsulfonamido) acetamide, a preexisting MMP-12 inhibitor (Figure 5).

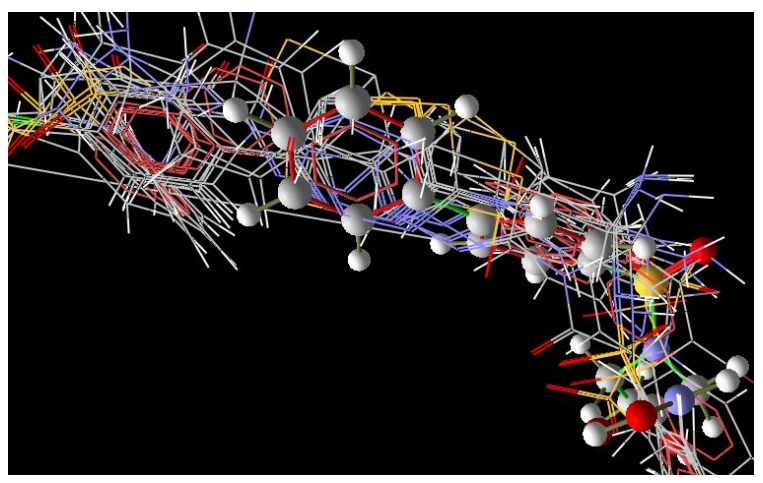

Figure 5. Superimposed docking images of novel 6(H)-1,3,4-thiadiazine derivatives (showing with wireframe model) with the pre-existing ligand (N-hydroxy-2-(N-hydroxyethyl)biphenyl-4-ylsulfonamido) acetamide with the MMP-12 showing interacting amino acids (showing with ball and stick model)

\section{Conclusion}

Computational study of novel 6(H)-1,3,4-thiadiazine derivatives proved them potential human MMP12 inhibitors. Although a systemic biochemical study is necessary to confirm the findings. On comparing the chemical structure of $6(\mathrm{H})-1,3,4$-thiadiazine derivatives with (N-hydroxy-2-(N-hydroxyethyl) biphenyl-4ylsulfonamido) acetamide, a pre-existing human MMP12 inhibitor; it is concluded that a bicyclic system, a sulfonamido linkage and a acetamide functionality are essential pharmacophoric requirements in designing of human MMP12 inhibitors.

\section{References}

[1] Verma, R.P.,Hansch, C., "Matrix metalloproteinases (MMPs): Chemical-biological functions and (Q)SARs," Bioorg. Med. Chem 15, 2223-2268, 2007.

[2] Belaaouaj A, Shipley J.M., Kobayashi D.K., Zimonjic D.B., Popescu N., Silverman G.A., Shapiro S.D., "Human macrophage metalloelastase. Genomic organization, chromosomal location, gene linkage, and tissue-specific expression," J. Biol. Chem, 270 (24), 14568-14575, 1995.

[3] Verma, R.P.,Hansch, C., "QSAR modeling of taxane analogues against colon cancer," Eur. J. Med. Chem. 45, 1470-1477, 2010.

[4] Sardana, S.,Madan, A.K., "Topological models for prediction of antihypertensive activity of substituted benzylimidazoles," $J$. Mol.Struct, 638, 41-49, 2003.

[5] Gregg, S.,Eiso, A.B., Jan, S., "Integration of fragment screening and library design," Drug Discovery Today, 12(23/24), 1032-1039, 2007.

[6] Abraham, H. M., Ibrahim, A.,Zissimos, M. A., "Determination of sets of solute descriptors from chromatographic measurements.," Jour. Chromat, 1037, 29-47, 2004

[7] Hou, T.J.,Xu X.J., "ADME Evaluation in Drug Discovery. 3. Modeling Blood-Brain Barrier Partitioning Using Simple Molecular Descriptors," J. Chem. Inf. Comput. Sci, 43, 2137-2152, 2003.

[8] Sharma, J.,Ramanathan, K.,Rao, S., "Identification of Potential Inhibitors against Acetylcholinesterase Associated With Alzheimer's Diseases: A Molecular Docking Approach," $J$. Comput. Method Mol. Design, 1(1), 44-51, 2011.

[9] Yap, C.W., 2011. "PaDEL-descriptor: An open source software to calculate molecular descriptors and fingerprints," J. Comput. Chem. 32(7), 1466-1474, 2011. 